\title{
Nano-opto-electronics for biomedicine
}

\author{
GU Ning*, LI Yan, WANG Meng \& CAO Min \\ State Key Laboratory of Bioelectronics, Jiangsu Key Laboratory of Biomaterials and Devices, School of Biological Science and \\ Medical Engineering, Southeast University, Nanjing 210096, China
}

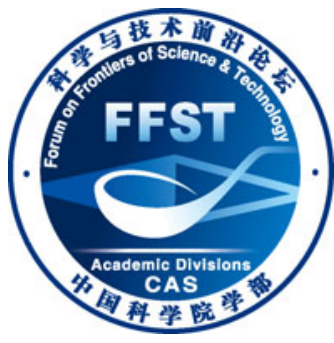

Received February 26, 2013; accepted April 24, 2013; published online June 5, 2013

\begin{abstract}
Nano-opto-electronics for biomedicine is a developing interdisciplinary field. Related areas of this field are tried to be classified and outlined herein. Progresses in the different areas were reviewed based on the research development of the invited experts. Prospects of nano-opto-electronics for biomedicine are discussed.
\end{abstract}

photonics, optoelectronics, biomedicine, nanomaterials

Citation: $\quad$ Gu N, Li Y, Wang M, et al. Nano-opto-electronics for biomedicine. Chin Sci Bull, 2013, 58: 2521-2529, doi: 10.1007/s11434-013-5917-9

Recently, Nano-technology for biomedical research has received extensive attention, and considerable progress has been made in this area. Nano-tech encompasses many advanced research areas, such as nano-characterization and nano-measurement based on opto-electric technology, nanomaterial with optically modulated parameters, nano-photonics and nano-optoelectronics, etc. These research areas have demonstrated important applications such as detection and manipulation of nano bio-structure, in vitro and in vivo characterization of bio-molecule for clinical diagnosis, imaging and detection of single cells and cell masses and tissues, bio-effect of functional nano-material for dietetic therapy, and nano-transportation system of medicine. In brief, nano-technology for bio-medical research has developed into an important and highly promising interdisciplinary area. The development of related technologies is summarized in Figure 1.

As the Chief Editor of "Chinese Science Bulletin", Prof. Xia Jianbai has organized many symposia in this area. Some researchers in this field were invited to present their latest works, discuss future prospective developments and look for possible collaborations. According to these discussions and suggestions from Prof. Xia, we write this paper. We try to organize the development theme of related areas, and try

*Corresponding author (email: guning@ seu.edu.cn)

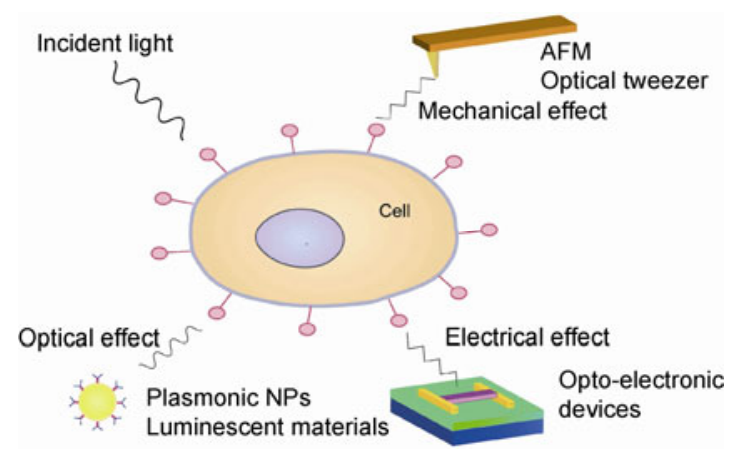

Figure 1 (Color online) Illustration of nano-opto-electronics for biomedicine.

to classify different areas of nano-photonics and nanooptoelectronics in bio-medical research based on progresses in Chinese and international researchers, especially the important achievements by invited experts in this edition, and try to discuss future developments.

\section{Nano-scale characterization and measure- ment of biological objects based on atomic force microscopy (AFM)}

With the development of opto-electric technology, the nanoscale characterization and measurement technology has 
been greatly promoted. Some of these methods could be successfully applied in the fields of biology and medicine. Here, as an example, the basic principle and main applications of AFM are briefly introduced.

The atomic force microscope, one type of scanning probe microscopes, has been invented as a variation of the scanning tunnel microscope with the development of several related technologies including optoelectronic technology, precision machining, weak signal detection and processing technique, etc. The basic idea of AFM is to use a sharp tip scanning over the surface of the sample while sensing the interaction between the tip and the sample [1]. The sharp tip is fixed at the end of a flexible cantilever. The cantilever senses forces acting between the tip and the sample surface causing its deflection. The signal is usually recorded by the optical system: the laser beam is focused at the cantilever end and then, after reflection, is detected by a position sensitive photodiode. The AFM system senses these changes in position and can map surface topography or monitor the interaction force between the tip and the sample.

One of the most common applications of AFM in biomedicine is to visualize morphology of biological objects ranging from single molecules such as DNA, RNA, proteins, single cells, to tissues and organs [2]. Combinations of AFM with other complementary techniques provide great opportunities for obtaining more comprehensive information from interesting samples, which further expands AFM applications in biomedicine. These techniques include fluorescent microscopy, confocal microscopy and Raman spectroscopy [1,3]. The combination of AFM topography and fluorescence images provides a unique tool for correlating organelles and chemical components with cell morphology. Various fluorescent dyes used to selectively label the organelles and molecular complexes have been reported. A new star-shaped conjugated oligoelectrolyte used for direct imaging in living PANC-1 cells was reported in this issue [4]. The coupling of Raman spectroscopy to scanning probe microscopy has opened new perspectives on the subject of requiring both topography and chemical information with high spatial resolution. Tian's group [5] has reported important works on tip-enhanced Raman spectroscopy.

In addition to morphology imaging, AFM can also be used in force spectroscopy mode, which allows the detection and manipulation of single molecules, providing novel insight into their structure-function relationships. In the force spectroscopy mode, the cantilever approaches the sample at a given spatial position, contacts it, and when it reaches the maximum deflection value, it is withdrawn backward. The cantilever deflection (i.e. force signal) is recorded as a function of its vertical displacement (i.e. distance signal) as the tip approaches toward and retracts from the sample to obtain a force-distance curve. Moreover, spatial resolution can be achieved by generating a force-volume image through acquiring force-distance curves at multilocations. The record contains the information about inter- action force and physical properties of the sample. The applicability of AFM to studies of cancer transformation, based on the mechanical properties of living cells, was presented in the 2nd National Conference on Nanotechnology "NANO 2008" [6]. The cell's ability to deform and to adhere seems to describe the overall changes occurring in the cell structure and interaction forces introduced by cancer transformation. In this report, it was considered that the cell deformation (i.e. cell stiffness) could be usually quantified by the Young's modulus and the unbinding force determined the adhesive properties of a cell. Thus, the quantitative analysis of cell deformability and the expression of adhesion proteins between normal (reference) cells and pathologically changed cells (e.g. cancerous cells) have a significant impact on the quantitative description of characteristics for cancerous cells and further the cancer detection on a single cell level. Several important studies about mechanical properties measurement of cancerous cells via AFM have also been demonstrated in the paper. In vitro assay of cytoskeleton nanomechanics was used as a tool for screening potential anticancer effects of natural plant extract, tubeimoside I on human HepG2 cells by Deng's group [7] in this issue.

Since its invention, the ability of this microscope to achieve high resolution (subnanometer) imaging in liquids and to probe the mechanical properties of the sample at a nanometric scale makes this instrument increasingly useful for the study of biological specimens. AFM was employed as a powerful tool to explore morphology and ultrastructure of Hela cells treated with cinobufacini and curcumin by Cai's group in this issue [8]. The history and milestones of application of AFM in biological science are today well presented in books and even textbooks [9]. Meanwhile, wonderful reviews on the biomedical application of AFM were also published [1,6,10,11]. Interested readers can refer to these references, which provide useful technical analyses and research information obtained in different categories, varied from determining high-resolution structure, probing mechanical properties and determining force interactions, to monitoring biological processes and dynamics.

\section{Trapping and manipulation of biological ob- jects based on optical tweezers technique}

The developments of optical tweezers have driven much of the current single molecule research and have opened exciting avenues of research, especially in biology. It could be successful that trapping of viruses, bacteria and eukaryotic cells, as well as the manipulation of organelles within cells, introducing the "optical tweezers" into the field of biological micromanipulation.

Optical tweezers are photonic devices that exploit a tightly focused laser beam to manipulate the dielectric particles in three dimensions (3D) in a noninvasive manner. 
Different from conventional mechanical system, the optical tweezers can tweeze, manipulate and fabricate small "work pieces" such as biological cells, organelles and other micro-particles by light. It is a remote micro-process without any mechanical touch. The technique of optical tweezers and manipulation of small neutral particles by lasers is based on the forces of radiation pressure. These forces arise from the momentum of the light itself. The physical principles of optical trapping have been described in detail [12]. Briefly, the cell is pulled laterally into the center of the beam due to the transverse intensity gradient of the Gaussian laser beam. For a spot diameter on the order of the wavelength of light, the force due to the axial laser beam gradient pointing towards the beam waist is greater than the scattering force in the direction of the light. This pulls the cell vertically to a point just blow the laser beam waist, thereby completing the $3 \mathrm{D}$ trap. With the choice of appropriate laser wavelength and the power used in optical trapping experiments, there is no visible damage to the living cells.

It was first documented by Ashkin [13] in 1970 that optical forces, or radiation pressure, could be used to trap and accelerate dielectric polarizable micron-sized particles. For a good overview on the evolution of optical trapping, a review from Ashkin [14] is recommended. After that, experiment with optical tweezers, which are used for holding and manipulating a variety of nanometer- to micron-sized biological objects such as DNA, single molecules, membrane bilayers, intracellular structures, vesicles, and plant cells, were reported [15].

One specific application of optical tweezers is optical force measurements, which may display the biological objects' mechanical properties such as elasticity, stiffness, rigidity, torque, and dynamic behavior. By measuring the displacement of a bead or cell from its equilibrium position in the trap, forces in the $\mathrm{pN}$ range can be measured. And this is adequate to investigate a large variety of phenomena in both molecular and cell biology. Exploring mechanochemical processes in the cell with optical tweezers has been reviewed early in 2006. The paper started by a short introduction of optical tweezers and its advantage for single molecule manipulations, followed by the physics for understanding optical trapping and the description of the optical tweezers setup. The experimental progress in molecule and cell biology achieved by optical tweezers was also illustrated [16].

To date optical tweezers are used in considerable amount of single cell experiments. Tan et al. [17] studied human red blood cell mechanical properties in relation to osmotic conditions by using optical tweezers to stretch single cells. In this study, finite element simulation was also performed to develop cell mechanical model to compare simulation data and cell-stretch experiment data. This study indicated that osmotic stress has considerable effect on RBC's mechanical properties. In order to study the relations between cell me- chanics and diseases, single cell mechanics of myeloblasts from AML patients was investigated using optical tweezers and microbeads as handles to stretch a single cell [12].

Apart from the use of itself, nowadays, optical tweezers is also combined with other analytical methods to form powerful tools in single molecule studies. Once the cell has been stabilized in the optical trap, various diagnostics can be performed with a higher signal-to noise ratio. This is the benefit of combined application of optical tweezers with other techniques. Among these techniques, optical imaging techniques, such as fluorescence, confocal and multiphoton microscopy $[18,19]$, are used most commonly because optical trapping can preferably be performed with the same microscopy objective as is used for imaging of the sample. Besides optical microscopy, Raman spectroscopy could be used simultaneously with optical tweezers, which has been extensively studied, with some promising applications including tumor identification and cell surgery for organelle extraction. Raman spectroscopy is well suited for use with optical tweezers systems simultaneously because both techniques require a very tightly focused beam for optimal performance. For example, by combining Raman spectroscopy with traditional fluorescence imaging into the same optical trapping setup, it is possible to trap the cell while acquiring the Raman signature as well as fluorescence emission spectrum and simultaneously imaging the cell [20]. In this issue, the identification of different type of single cells in aqueous medium and discrimination the sources of same type cells from different donor using a novel technique which combined laser trapping and micro-Raman spectroscopy is reported by Ye's group [21].

Further more, enhanced cell sorting and manipulation with combined optical tweezer and microfluidic chip technologies was demonstrated [22]. In the study, a unique cell sorting and manipulating device by combining microfluidic chip and optical tweezer was designed. The cells were focused to desired area by microfluidics flow, recognized by image processing, and precisely moved by optical tweezers. This device had three unique properties: high purity and high recovery time, multi-cell sorting can be achieved, and multiple features of cells can be tracked.

Although the task of characterizing molecular machines and organelles seems daunting, there has been exciting progress. In the near future, scientists may come to see each cell as an individual with its own set of molecular machinery. By using methods like optical tweezers for manipulating single molecules, biologists will be able to investigate the nature of molecular machines one by one, and infer from their behavior those properties common to the population [23].

\section{Compact nano-optoelectronic devices}

Micro/nano electromechanical systems (MEMS/NEMS) 
refer to nanoscopic devices integrating electrical and mechanical functionality on the nanoscale. Combination of MEMS/NEMS technologies and optoelectronics is expected to have a major impact in healthcare, medicine and the life sciences through the creation of powerful tools for improved diagnosis and treatment of disease [24]. In biomedicine, there is a requirement for the development of medical devices for the detection of physical and chemical parameters in the human body. MEMS/NEMS offers great potential advantages over other types of conventional medical devices due to their small size scale, electrical nature, and ability to operate on short time scales [25]. The application of MEMS/NEMS based devices enable rapid, sensitive, and real time analysis of biological and chemical species, and is attracting increasing interest in the scientific community. Semiconductor nanomaterials, including nanotubes (NTs), nanowires (NWs) and graphene, are important transducer materials for MEMS/NEMS due to their remarkable physical and mechanical properties, such as high current-carrying capability and mechanical strength [26].

Semiconductor NTs and NWs are direct-bandgap materials, which can be configured either as field-effect transistor (FETs), light emitters or light detectors. NTs/NWs have been demonstrated to be configured as field-effect transistors (FETs), which are suitable candidates for designated biosensors [27]. A NW/NT FET is a three-terminal switching device, including source, drain, and gate electrodes. The transistor conduction is controlled by an applied voltage at the gate insulator. The adsorption of charged biomolecules such as DNA, RNA, and proteins onto the NT/NW surface produces a change in the electrical field, resulting in the change in the current flow through the device [28]. Thus, FET based biosensors hold the promise of detecting single-molecule events. In this issue, an AlGaN/GaN heterojunction field-effect transistor (HFET) biosensor was designed for real time electrical detection of single-stand DNA (ssDNA) hybridization by Lu's group [29].

In the last two decades, a diversity of FET based biosensors has been employed for biological applications, such as protein-protein interactions, DNA hybridization, and enzymatic glucose detection [28]. FET based biosensors exhibit several advantages over conventional macroscopic biosensors: label-free, real-time, high sensitivity and selectivity. However, it is extremely difficult to control the alignment and assembly for a FET base on a single NT or NW. An alternative strategy is the use of random network of NTs or NWs. Compared with a FET biosensor made from a single NT or NW, a random network FET is much easier to fabricate and is more robust because its characteristics are averaged over a large number of nanotubes. For example, Chen et al. [30] reported the detection of antibodies and proteins by NT random network, where the nonspecific binding on nanotubes is overcome by immobilization of polyethylene oxide chains.

Real time intracellular monitoring is important in many biomedical studies. However, planar FET-based devices are not feasible for in vivo monitoring. Lieber's group [31] reported a 3D nanoscale FET device with kinked NW structures which can enter single cells to record intracellular potentials, functioning as a patch-clamp micropipette. Subsequently, they reported a new approach in which a silica NT is synthetically integrated on top of a nanoscale FET [32]. This nanotube penetrates the cell membrane, bringing the cell cytosol into contact with the FET, which is then able to record the intracellular potential. These studies are expected to open up opportunities for intracellular monitoring and chemical sensing.

Single-walled carbon nanotubes (SWNT) can also be utilized as optical biosensors due to their ability to excitation photoluminescence arising from the electronic band gap. The band-gap energy is sensitive to the local dielectric environment around the SWNT. DNA can bind to the surface of SWNTs through $\pi$-stacking, and directly modulate the transient energy shift of the SWNT fluorescence. The DNA wrapped nanotubes (DNA-SWNTs) have been used to monitor DNA hybridization, and biologically relevant analyses [33]. Furthermore, SWNT fluoresce within the tissue transparency window, providing new opportunities for optical sensors that operate in strongly absorbing media of relevance to medicine or biology.

MEMS/NEMS also offers significant advances in drug delivery because of their digital capabilities and short response times. Furthermore, incorporation of sensing components may allow for the creation of an intelligent MEMS/ NEMS drug delivery device. A variety of drug delivery devices based upon MEMS/NEMS technology, including devices based on microporous, silicon, microneedles, micropumps, and microreservoirs have been proposed [34]. A typical example is silicon-based drug delivery device consisting of an array of microreservoirs. The microreservoir in the device is covered with a gold membrane, containing a dosage of drug. Application of a potential to a gold membrane results in electrochemical dissolution of the membrane, allowing the drug within the reservoir to dissolve and diffuse into the surrounding medium. Each microreservoir can be individually filled, enabling the pulsatile release of multiple substances [35].

Optical fiber probe sensor is also an important nanooptoelectronic device. In this issue, Zhu's group [36] has fabricated a selectable infiltrating large hollow core photonic band-gap fiber, which has potential application for implementing novel lasers, sensors and tunable optoelectronic devices. Guo's group [37] developed a high-sensitive and temperature-self-calibrated tilted fiber grating sensing probe, by using which, human acute leukemia cells with different intracellular densities could be clearly discriminated through their refraction index (RI) measurement, to study the relationship between the intracellular density of cells and their RI. 


\section{Surface plasmon resonance}

Over the past decades, metal nanoparticles have attracted significant attention due to their unique localized surface plasmon resonance (LSPR) properties, which originate from the collective oscillation of conduction electrons in response to optical excitation. The resonance creates enhanced light scattering and absorption and large local field enhancement, strongly depending on the shape, composition, and arrangement of the particles, and the properties of local environment. By changing these factors, one can control the design of desired nanomaterials for applications in biological sensing [38,39], medical imaging and diagnostic [40,41], and photothermal therapy [42].

The fact that LSPR is sensitive to the local environment of the nanoparticle surface has been exploited for a range of biological sensing strategies. For example, a receptor-functionalized gold nanoparticle will respond to the bonding of the target molecules through a shift in the resonance frequency. This change signals the presence of target molecules in the optical spectrum of the nanoparticle. To ensure high analyte sensitivity, distinct changes in the LSPR peak position in response to relatively small changes in the medium refractive index are desire. Many theoretical and experimental studies have been carried out on metal nanoparticles with different geometry to find the best nanoparticle configuration to enhance the sensitivity of the LSPR response [43-45]. Application of nanomaterials to SPR- based bio-detection and sensitivity improvement of LSPR has also been described in the article in this issue [46].

The enhanced scattering cross sections of metal nanoparticles have been utilized for optical imaging and labeling of biological systems using techniques such as dark-field microscopy, optical coherence tomography and laser reflectance confocal microscopy [47-49]. Compared with fluorescent labels, metal nanoparticles have advantages as the particle scattering cross section is three to five orders of magnitude greater than the fluorescence cross section of a single molecule [50]. This principle was for the first time employed by El-Sayed's group [51] for their new method of simple and reliable diagnosis of cancer with the use of gold nanoparticles.

The strong light absorption followed by rapid photothermal conversion of metal nanoparticles has been used for selective damage to target cancer cells. When irradiated with a laser of frequency overlapping with the LSPR absorption maximum of the particles, the photothermal heating of the medium surrounding the nanoparticle can be achieved. In addition, metal nanoparticles can keep their optical properties in cells for a long time under certain conditions. Successive irradiation with several laser pulses allows control of cell inactivation by a nontraumatic means [52].

The plasmon field enhanced Raman and fluorescence signals of molecules in the vicinity of metal nanoparticles have been recently utilized for the molecular diagnosis of cancer [53]. For example, gold nanoparticles conjugated with anti-EGFR antibodies assembled onto the surface of cancer cells due to specific binding, resulting in the observation of highly enhanced, sharp, and polarized SERS of the nanoparticle capping and bridging molecules, anti-EGFR, and EGFR receptors or other molecules on the cell surface [54].

\section{Light-emitting or luminescent nanomaterials, nanomaterials with optical conversion properties and their biomedical applications}

For a long time, extensive research has been focused on the development of various methods to precisely control the synthesis of light-emitting or luminescent nanomaterials, and nanomaterials with optical conversion properties. Many different kinds of nanostructures were designed and synthesized, including quasi-zero dimensional nanodots, quasi-one dimensional nanowires, nanosheets and other complicated nanostructures, which are considered to be promising building blocks for electrical, optical and optoelectronic applications. And the development of these nanostructures promotes the construction of new opto-electronic nanodevices, functional nanosystems, and eventually, the development of the related instruments and technologies as mentioned in the above sections of this paper. The recent advances in research on these different kinds of nanostructures, focusing on their synthesis, surface functionalization, properties, and applications were reviewed in a few publications during the past few years. For example, nanostructured $\mathrm{ZnO}$ and gold nanorods, see also in the reviews of Xu's group [55] and Zhao's group [56] in this issue. We just try to outline the typical related nanostructures and their biomedical applications herein.

During the last two decades, a great deal of attention has been focused on the optoelectronic properties of nanostructured semiconductors. If the extent of the material is on the order of one to ten nanometers in all three directions, the material is said to be a quantum dots (QDs). Semiconductor QD nanocrystals, which are usually be categorized according to the columns in the periodic table, for example III-V materials ( $\mathrm{GaN}$, InP, etc.), II-VI materials ( $\mathrm{ZnO}, \mathrm{CdS}$, $\mathrm{CdTe}$, etc.) and IV-VI materials (PbS, PbTe, etc.) [57], are perhaps one of the most ubiquitous optical nanoprobes due to their excellent photostability, large luminescence quantum yield as well as their size-tunable absorption and emission wavelengths [58]. In the past years, tremendous research efforts have been devoted to producing high quality QDs by optimizing synthetic procedures, functionalizing the QD surface to enhance biocompatibility, and coupling the QDs to agents with complementary functions (e.g. targeting molecules of therapeutic agents $[59,60]$. The development of a wide range of methods for bio-conjugating QDs in 
diverse areas of application: cell labeling [61], cell tracking [62], in vivo imaging [63], DNA detection [64], and multiplexed beads [65] have been seen. In this issue, the effect of protein molecules on the photoluminescence properties and stability of the water soluble CdSe/ZnS QDs coated with amphiphilic PAA is reported by Kong's group [66]. A simple, sensitive and rapid acetylcholine esterase (AChE) measuring strategy based on the CdTe QDs have been constructed by Tang's group [67]. And Li's group [68] developed a novel method to fabricate derivatized Tween-coated silica beads for protein detection. Under the optimized experimental conditions, the detection limit of rabbit IgG using sandwich immune assay is as low as $50 \mathrm{pg} / \mathrm{mL}$, which is sufficiently low for the current methods.

Even though optical techniques continue to evolve, the fact is that electrical detection remains extremely desirable. Electrical systems can be miniaturized and integrated into synstems, offering many advantages over optical detection schemes. Pseudo-1 dimensional nanostructures, such as semiconductor nanowires and carbon nanotubes offer the greatest chance yet for creating robust, sensitive, and selective electrical detectors of biological binding events, which is discussed in the section above.

Highly fluorescent carbon nanomaterials called carbon dots (CD) have drawn increasing attention owing to their attractive applications in optoelectronic devices and biomedical imaging. Compared with conventional organic dyes and semiconductor QDs, photoluminescent CDs are superior in terms of chemical inertness, large two-photon excitation cross-sections, absence of blinking, low cytotoxicity, and excellent biocompatibility. During the past few years, much progress has been achieved in the synthesis, property and applications of CDs, as recently reviewed [69,70]. For example, a novel CD synthetic process with high optical quantum yield $(47 \%)$ with fast turnaround (main reactions finish in $1 \mathrm{~min}$ ) was demonstrated, which made a breakthrough in CD fabrication [71]. A graphene oxide fluorescence switch strategy was designed to monitor the RNA transcription levels sensitively by Xing's group [72] in this issue. Due to its large surface area, superior mechanical flexibility, high carrier transport mobility and good thermal/chemical stability [73], graphene are suitable for using as optoelectronic devices [74]. Especially, the high mobility of graphene renders it a promising electrode and electronacception material for photovoltaic device applications [75].

Another class of potentially non-toxic and biocompatible fluorescent nanoparticles is those of silicon (silicon dots-SD). Since they display photoluminescent properties reminiscent of those of QDs [76,77], CD and SD have already demonstrated their viability in a variety of applications including bioimaging, peroxidase mimetics, photodynamic therapy, etc. Moreover, a breakthrough in gene therapy research was also made by designing and synthesizing a fluorescent cationic poly fluorine (CCP), achieving 92\% delivery and transfection efficiency for pDNA (plasmid
DNA) encoding GFP (green fluorescent protein), outperforming commercial transfection agents. This CCP also facilitates tracking due to its excellent fluorescent and optical stability property [78].

Another important nanostructured material in opto-electronics for bio-medicine is metallic nanostructures. The electronic and optical properties of metals depend greatly on their size, in particular in the nanometre range. One important property of metal nanoparticles is surface plasmon resonance (SPR), which is described in other section. Besides metal nanoparticles of larger size, fluorescent metallic nanoclusters (NCs) consist of several to a hundred atoms, focusing on $\mathrm{Au}$ and $\mathrm{Ag} \mathrm{NCs}$, has also been developed for biomedical applications. A distinct feature of NCs is its strong photoluminescence, combined with good photostability, large Stokes shift and high emission rates. Moreover, the advances in synthesis of water-soluble fluorescent NCs with different ligands and tunable emission colors in various biocompatible scaffolds, render them an ideal fluorophores for applications as biological labels or optoelectronic emitters [79]. Cell imaging is no doubt one application of fluorescent $\mathrm{Au}$ and $\mathrm{Ag}$ nanostructures. In this issue, Han's group [80] reported a multiple labeling method for cells, by introduing $\mathrm{Au}$ nanooctahedra and $\mathrm{Au}$ nanorods as biomarkers for ICAM-1 and Integrin B1, different molecules on cell surface. And fluorescence imaging using $\mathrm{Au}$ and $\mathrm{Ag}$ nanostructures conjugated with different molecules has been reported elsewhere. Various biopolymers (proteins and DNA) can also been detected using Au and Ag NCs [81]. For example, polyclonal, goat-derived anti-human IgG antibody-modified PAMAM-Au NCs have been used for the detection of the human IgG antigen [82]. Some reviews on the preparation and applications of fluorescent gold and silver NCs have also appeared $[79,83]$. And the recent progress of gold nanorods in bioimaging, drug delivery, diagnostics and photothermal therapy for disease treatment was also described by Zhao's group in this issue [56].

The luminescence of all the fluorescent nanostructures mentioned above relies on energy down-conversion, thus have to be excited either by UV, potentially harmful and carcinogenic, or blue-green visible radiation [84]. Lanthanide-doped up-converting nanocrystals may be a promising alternative because lanthanide ions have the ability to efficiently up-convert near-infrared radiation into shorter wavelengths [85]. Recently, rare-earth-doped nanoparticles demonstrated their great potential in many fields of biological science including cell and tissue labeling for bioimaging, biodetection, therapy or multiplexed analysis [86-88].

\section{Future of nano-opto-electronics for biomedicine}

Based on the detailed introductions of these related fields, the future of nano-opto-electronics for biomedicine can be 
clearly prospected, which is addressed as follows:

(1) Bio-effects of nano-optical materials, including their effects on bio-object (specific cells and tissues), and their application in biological processes.

(2) Assembly and formation of structures by bio molecules on nano-particles, modulation of optical characteristics of nano-particles and its application to bio-molecules detection.

(3) On-line higher resolution and faster speed imaging of cells (molecules) by using optical, opto-acoustic and X-raybased techniques.

(4) Detection and manipulation of molecules and cells by SPM, optical tweezers and related technologies. Furthermore, it is urgently needed to reveal and understand biological structures and characterize their developing process on nanometer scale from various angles.

(5) Plasmonics becomes a more and more important research area, involving not only nano-materials and structure, but also opto-electric devices and systems. As a consequence, bio-detection methods based on new principles would be developed, such as nano-lasers and its application to bio-medicine. Relevant theoretical models should be built up.

(6) MEMS application has been widely adopted, particularly in biological and medical detection devices and systems. Among these applications, opto-electric detections employing polymer and organic opto-electric materials are especially important, such as MOMES (micro-optical mechanic electronic system). The biocompatibility in the process needs more attention. NEMS (nano electrical and mechanic system) are also becoming attractive research areas.

(7) Nanomaterials with optical conversion properties, such as opto-thermal conversion materials, have been successfully implemented in in vivo transport of medicine, physical therapy of cancer. Further studies on their biological safety and relevant medical technology need to be carried out before clinical practice.

(8) Bio-medical applications based on novel opto and opto-electric devices.

This work was supported by the National Basic Research Program of China (2011CB933503).

1 Liu S, Wang Y. Application of AFM in microbiology: A review. Scanning, 2010, 32: 61-73

2 Kasas S, Thomson N H, Smith B L, et al. Biological applications of the AFM: From single molecules to organs. Int $\mathrm{J}$ Imaging Syst Technol, 1997, 8: 151-161

3 Flores S M, Toca-Herrera J L. The new future of scanning probe microscopy: Combining atomic force microscopy with other surfacesensitive techniques, optical and microscopy fluorescence techniques. Nanoscale, 2009, 1: 40-49

4 Song W L, Jiang R C, Yuan Y, et al. Star-shaped conjugated oligoelectrolyte for bioimaging in living cells. Chin Sci Bull, 2013, 58: 2570-2575

5 Liu Z, Ding S Y, Chen Z B, et al. Revealing the molecular structure of single-molecule junctions in different conductance states by fishing-mode tip-enhanced Raman spectroscopy. Nat Commun, 2011, 2: 305-308

6 Lekka M, Wiltowska-Zuber J. Biomedical applications of AFM. In: Kolodziej J, Konior J, Czuba P, et al., eds. Nano 2008: 2nd National Conference on Nanotechnology, 2008 Jun 25-28, Cracow, Poland. J Phys Conference Ser, 2009, 146: 012023

7 Zhao H B, Wang Y S, Jiang X M, et al. In vitro assay of cytoskeleton nanomechanics as a tool for screening potential anticancer effects of natural plant extract, tubeimoside I on human hepatoma (HepG-2) cells. Chin Sci Bull, 2013, 58: 2576-2583

8 Liu L, Jin H, Ou J L, et al. Cinobufacini-induced hela cell apoptosis enhanced by curcumin. Chin Sci Bull, 2013, 58: 2584-2593

9 Braga P C, Ricci D. Atomic Force Microscopy: Biomedical Methods and Applications. Totowa: Humana Press, 2004

10 Dorobantu L S, Goss G G, Burrell R E. Atomic force microscopy: A nanoscopic view of microbial cell surfaces. Micron, 2012, 43: 1312-1322

11 Wright C J, Shah M K, Powell L C, et al. Application of AFM from microbial cell to biofilm. Scanning, 2010, 32: 134-149

12 Youhua $\mathrm{T}$, Leung A Y H, Kaiqun W, et al. Optical tweezer technology. IEEE Nanotechnol Mag, 2011, 5: 17-21

13 Ashkin A. Acceleration and trapping of particles by radiation pressure. Phys Rev Lett, 1970, 24: 156

14 Ashkin A. Optical trapping and manipulation of neutral particles using lasers. Proc Natl Acad Sci USA, 1997, 94: 4853-4860

15 Moffitt J R, Chemla Y R, Smith S B, et al. Recent advances in optical tweezers. Annu Rev Biochem, 2008, 77: 205-228

16 Hormeno S, Arias-Gonzalez J R. Exploring mechanochemical processes in the cell with optical tweezers. Biol Cell, 2006, 98: 679-695

17 Tan Y H, Sun D, Wang J Z, et al. Mechanical characterization of human red blood cells under different osmotic conditions by robotic manipulation with optical tweezers. IEEE Trans Biomed Eng, 2010, 57: $1816-1825$

18 Goksor M, Enger J, Hanstorp D. Optical manipulation in combination with multiphoton microscopy for single-cell studies. Appl Optics, 2004, 43: 4831-4837

19 Enger J, Goksor M, Ramser K, et al. Optical tweezers applied to a microfluidic system. Lab Chip, 2004, 4: 196-200

20 Butler C, Fardad S, Sincore A, et al. Multispectral optical tweezers for molecular diagnostics of single biological cells. In: Farkas D L, Nicolau D V, Leif R C, eds. Conference on Imaging, Manipulation, and Analysis of Biomolecules, Cells, and Tissues X, 2012 Jan 21-23, San Francisco, CA. Proc SPIE, 2012, 8225: 82250C

21 Ma H F, Zang Y, Ye A P. Single-cell discrimination based on optical tweezers Raman spectroscopy. Chin Sci Bull, 2013, 58: 2594-2600

22 Wang X L, Chen S X, Kong M, et al. Enhanced cell sorting and manipulation with combined optical tweezer and microfluidic chip technologies. Lab Chip, 2011, 11: 3656-3662

23 Husale S C. Single biomolecule studies using optical tweezers. Dissertation for Doctoral Degree. Basel: University of Basel, 2005

24 Bhushan B. MEMS/NEMS and biomems/bionems: Materials, devices, and biomimetics. In: Bhushan B, ed. Springer Handbook of Nanotechnology. Heidelberg: Springer, 2010. 1663-1740

25 Grayson A R, Shawgo R S, Johnson A M, et al. A biomems review: MEMS technology for physiologically integrated devices. Proc IEEE, 2004, 92: 6-21

26 Avouris P, Freitag M, Perebeinos V. Carbon-nanotube photonics and optoelectronics. Nat Photonics, 2008, 2: 341-350

27 Yang W R, Ratinac K R, Ringer S P, et al. Carbon nanomaterials in biosensors: Should you use nanotubes or graphene? Angew Chem Int Ed, 2010, 49: 2114-2138

28 Chen K I, Li B R, Chen Y T. Silicon nanowire field-effect transistor-based biosensors for biomedical diagnosis and cellular recording investigation. Nano Today, 2011, 6: 131-154

29 Wen X J, Wang S N, Wang Y J, et al. AlGaN/GaN heterostructure field transistor for label-free detection of DNA hybridization. Chin Sci Bull, 2013, 58: 2601-2605

30 Chen R J, Bangsaruntip S, Drouvalakis K A, et al. Noncovalent 
functionalization of carbon nanotubes for highly specific electronic biosensors. Proc Natl Acad Sci USA, 2003, 100: 4984-4989

31 Tian B Z, Cohen-Karni T, Qing Q, et al. Three-dimensional, flexible nanoscale field-effect transistors as localized bioprobes. Science, 2010, 329: 830-834

32 Duan X J, Gao R X, Xie P, et al. Intracellular recordings of action potentials by an extracellular nanoscale field-effect transistor. Nat Nanotechnol, 2012, 7: 174-179

33 Heller D A, Jin H, Martinez B M, et al. Multimodal optical sensing and analyte specificity using single-walled carbon nanotubes. Nat Nanotechnol, 2009, 4: 114-120

34 Staples M, Daniel K, Cima M J, et al. Application of micro- and nano-electromechanical devices to drug delivery. Pharm Res, 2006, 23: $847-863$

35 Santini J T, Richards A C, Scheidt R, et al. Microchips as controlled drug-delivery devices. Angew Chem Int Ed, 2000, 39: 2397-2407

36 Liu J G, Du Y X, Zhu N H, et al. Selectable infiltrating large hollow core photonic band-gap fiber. Chin Sci Bull, 2013, 58: 2606-2610

37 Liu F, Guo T, Liu J G, et al. High-sensitive and temperature-selfcalibrated tilted fiber grating biological sensing probe. Chin Sci Bull, 2013, 58: 2611-2615

38 Anker J N, Hall W P, Lyandres O, et al. Biosensing with plasmonic nanosensors. Nat Mater, 2008, 7: 442-453

39 Mayer K M, Hafner J H. Localized surface plasmon resonance sensors. Chem Rev, 2011, 111: 3828-3857

40 Yong K T, Swihart M T, Ding H, et al. Preparation of gold nanoparticles and their applications in anisotropic nanoparticle synthesis and bioimaging. Plasmonics, 2009, 4: 79-93

41 Bardhan R, Lal S, Joshi A, et al. Theranostic nanoshells: From probe design to imaging and treatment of cancer. Accounts Chem Res, 2011, 44: 936-946

42 Zhang J Z. Biomedical applications of shape-controlled plasmonic nanostructures: A case study of hollow gold nanospheres for photothermal ablation therapy of cancer. J Phys Chem Lett, 2010, 1: 686-695

43 Cao M, Wang M, Gu N. Optimized surface plasmon resonance sensitivity of gold nanoboxes for sensing applications. J Phys Chem C, 2009, 113: 1217-1221

44 Zhang S P, Bao K, Halas $\mathrm{N}$ J, et al. Substrate-induced fano resonances of a plasmonic: Nanocube: A route to increasedsensitivity localized surface plasmon resonance sensors revealed. Nano Lett, 2011, 11: 1657-1663

45 Offermans P, Schaafsma M C, Rodriguez S R K, et al. Universal scaling of the figure of merit of plasmonic sensors. ACS Nano, 2011, 5: 5151-5157

46 Zhang X X, Han X F, Wu F G, et al. Nano-bio interfaces probed by advanced optical spectroscopy: From model system studies to optical biosensors. Chin Sci Bull, 2013, 58: 2537-2556

$47 \mathrm{Hu}$ M, Chen J Y, Li Z Y, et al. Gold nanostructures: Engineering their plasmonic properties for biomedical applications. Chem Soc Rev, 2006, 35: 1084-1094

48 Chen J, Saeki F, Wiley B J, et al. Gold nanocages: Bioconjugation and their potential use as optical imaging contrast agents. Nano Lett, 2005, 5: 473-477

49 Sokolov K, Follen M, Aaron J, et al. Real-time vital optical imaging of precancer using anti-epidermal growth factor receptor antibodies conjugated to gold nanoparticles. Cancer Res, 2003, 63: 1999-2004

50 Jain P K, Lee K S, El-Sayed I H, et al. Calculated absorption and scattering properties of gold nanoparticles of different size, shape, and composition: Applications in biological imaging and biomedicine. $\mathrm{J}$ Phys Chem B, 2006, 110: 7238-7248

51 El-Sayed I H, Huang X H, El-Sayed M A. Surface plasmon resonance scattering and absorption of anti-egfr antibody conjugated gold nanoparticles in cancer diagnostics: Applications in oral cancer. Nano Lett, 2005, 5: 829-834

52 Dykman L, Khlebtsov N. Gold nanoparticles in biomedical applications: Recent advances and perspectives. Chem Soc Rev, 2012, 41: 2256-2282

53 Qian X M, Peng X H, Ansari D O, et al. In vivo tumor targeting and spectroscopic detection with surface-enhanced raman nanoparticle tags. Nat Biotechnol, 2008, 26: 83-90

54 Jain $\mathrm{P} \mathrm{K}$, Huang X H, El-Sayed I H, et al. Noble metals on the nanoscale: Optical and photothermal properties and some applications in imaging, sensing, biology, and medicine. Accounts Chem Res, 2008, 41: 1578-1586

$55 \mathrm{Xu} \mathrm{C} \mathrm{X}$, Yang C, Gu B X, et al. Nanostructured $\mathrm{ZnO}$ for biosensing applications. Chin Sci Bull, 2013, 58: 2563-2566

56 Ma Z Y, Xia H X, Liu Y P, et al. Applications of gold nanorods in biomedical imaging and related fields. Chin Sci Bull, 2013, 58: 2530-2536

57 Kitai A. Luminescent Materials and Applications. West Sussex: John Wiley \& Sons Ltd, 2008

58 Michalet X, Pinaud F F, Bentolila L A, et al. Quantum dots for live cells, in vivo imaging, and diagnostics. Science, 2005, 307: 538544

59 Biju V, Itoh T, Ishikawa M. Delivering quantum dots to cells: Bioconjugated quantum dots for targeted and nonspecific extracellular and intracellular imaging. Chem Soc Rev, 2010, 39: 3031-3056

60 Zrazhevskiy P, Sena M, Gao X H. Designing multifunctional quantum dots for bioimaging, detection, and drug delivery. Chem Soc Rev, 2010, 39: 4326-4354

61 Wu X Y, Liu H J, Liu J Q, et al. Immunofluorescent labeling of cancer marker her2 and other cellular targets with semiconductor quantum dots. Nat Biotechnol, 2003, 21: 41-46

62 Parak W J, Boudreau R, Le Gros M, et al. Cell motility and metastatic potential studies based on quantum dot imaging of phagokinetic tracks. Adv Mater, 2002, 14: 882-885

63 Dubertret B, Skourides P, Norris D J, et al. In vivo imaging of quantum dots encapsulated in phospholipid micelles. Science, 2002, 298: $1759-1762$

64 Taylor J R, Fang M M, Nie S M. Probing specific sequences on single DNA molecules with bioconjugated fluorescent nanoparticles. Anal Chem, 2000, 72: 1979-1986

65 Han M Y, Gao X H, Su J Z, et al. Quantum-dot-tagged microbeads for multiplexed optical coding of biomolecules. Nat Biotechnol, 2001, 19: 631-635

66 Zhang Y L, Tu L P, Zeng Q H, et al. Effect of protein molecules on the photoluminescence properties and stability of water-soluble CdSe/ZnS core-shell quantum dots. Chin Sci Bull, 2013, 58: 26162621

67 Chen Z Z, Ren X L, Tang F Q. Optical detection of acetylcholine esterase based on CdTe quantum dots. Chin Sci Bull, 2013, 58: 2622-2627

68 Zhao Z, Li Z, Li J. Tween-modified suspension array for sensitive biomolecular detection. Chin Sci Bull, 2013, 58: 2628-2633

69 Baker S N, Baker G A. Luminescent carbon nanodots: Emergent nanolights. Angew Chem Int Ed, 2010, 49: 6726-6744

70 Shen J H, Zhu Y H, Yang X L, et al. Graphene quantum dots: Emergent nanolights for bioimaging, sensors, catalysis and photovoltaic devices. Chem Commun, 2012, 48: 3686-3699

71 Wang F, Xie Z, Zhang H, et al. Highly luminescent organosilanefunctionalized carbon dots. Adv Funct Mater, 2011, 21: 1027-1031

72 Zhou X M, Liao Y H, Xing D. Sensitive monitoring of RNA transcription levels using a graphene oxide fluorescence switch. Chin Sci Bull, 2013, 58: 2634-2639

73 Geim A K. Graphene: Status and prospects. Science, 2009, 324: 1530-1534

74 Novoselov K S, Geim A K, Morozov S V, et al. Electric field effect in atomically thin carbon films. Science, 2004, 306: 666-669

$75 \mathrm{Li} \mathrm{Y,} \mathrm{Hu} \mathrm{Y,} \mathrm{Zhao} \mathrm{Y,} \mathrm{et} \mathrm{al.} \mathrm{An} \mathrm{electrochemical} \mathrm{avenue} \mathrm{to} \mathrm{green-}$ luminescent graphene quantum dots as potential electron-acceptors for photovoltaics. Adv Mater, 2011, 23: 776-780

76 Alivisatos P. The use of nanocrystals in biological detection. Nat Biotechnol, 2004, 22: 47-52

77 Veinot J G C. Synthesis, surface functionalization, and properties of freestanding silicon nanocrystals. Chem Commun, 2006, 4160-4168

78 Feng X L, Lü F T, Liu L B, et al. A highly emissive conjugated 
polyelectrolyte vector for gene delivery and transfection. Adv Mater, 2012, 24: 5428-5432

79 Shang L, Dong S J, Nienhaus G U. Ultra-small fluorescent metal nanoclusters: Synthesis and biological applications. Nano Today, 2011, 6: 401-418

80 Zhang K, Feng J T, Sun Q M, et al. A multiple-labeling method for cells using $\mathrm{Au}$ nanoparticles with different shapes. Chin Sci Bull, 2013, 58: 2640-2645

81 Shiang Y C, Huang C C, Chen W Y, et al. Fluorescent gold and silver nanoclusters for the analysis of biopolymers and cell imaging. $\mathrm{J}$ Mater Chem, 2012, 22: 12972-12982

82 Triulzi R C, Micic M, Giordani S, et al. Immunoasssay based on the antibody-conjugated pamam-dendrimer-gold quantum dot complex. Chem Commun, 2006, 5068-5070

83 Choi S, Dickson R M, Yu J H. Developing luminescent silver nanodots for biological applications. Chem Soc Rev, 2012, 41: 18671891

84 Wang F, Chatterjee D K, Li Z Q, et al. Synthesis of polyethylenimine/nayf4 nanoparticles with upconversion fluorescence. Nanotechnology, 2006, 17: 5786-5791

85 White K A, Chengelis D A, Gogick K A, et al. Near-infrared luminescent lanthanide mof barcodes. J Am Chem Soc, 2009, 131: 18069-18071

86 Bouzigues C, Gacoin T, Alexandrou A. Biological applications of rare-earth based nanoparticles. ACS Nano, 2011, 5: 8488-8505

87 Wang M, Abbineni G, Clevenger A, et al. Upconversion nanoparticles: Synthesis, surface modification and biological applications. Nanomed Nanotechnol Biol Med, 2011, 7: 710-729

88 Xie X J, Liu X G. Photonics upconversion goes broadband. Nat Mater, 2012, 11: 842-843

Open Access This article is distributed under the terms of the Creative Commons Attribution License which permits any use, distribution, and reproduction in any medium, provided the original author(s) and source are credited. 